\title{
The correlation functions of the regular tetrahedron and octahedron
}

\author{
Salvino Ciccariello \\ Università di Padova, Dipartimento di Fisica G. Galilei \\ Via Marzolo 8, I-35131 Padova, Italy \\ salvino.ciccariello@unipd.it
}

July 10, 2014

\begin{abstract}
The expressions of the autocorrelation functions $(\mathrm{CF})$ of the regular tetrahedron and the regular octahedron are reported. They have an algebraic form that involves the arctangent function and rational functions of $r$ and $\left(a+b r^{2}\right)^{1 / 2}, a$ and $b$ being appropriate integers and $r$ the distance. The $\mathrm{CF}$ expressions make the numerical determination of the corresponding scattering intensities fast and accurate also in presence of a size dispersion.
\end{abstract}

Synopsis: One reports the algebraic expressions of the tetrahedron's and octahedrons' correlations functions.

Keywords: Correlation functions, regular tetrahedron and octahedron, small-angle scattering 


\section{Introduction}

The algebraic knowledge of the autocorrelation function (CF) of a homogeneous particle, with a given shape, is useful from an applied point of view because polydisperse analyses with such a geometrical shape no longer require a numerical integration to derive the particle form factor. Of course few geometrical shapes have algebraic CFs. Up to now it has been shown that the CFs of the sphere (Guinier \& Fournet, 1955), the cube (Goodisman, 1980) and the right parallelepiped (Gille, 1999) only have an algebraic form. Those of the rotational cylinder (Gille,1987) and ellipsoid (Burger \& Ruland, 2001) do not have this property since they involve non-elementary transcendental functions, as the elliptic ones. For a recent review, see Gille (2013). Recently it has been reported that two more Platonic solids have an algebraic chord-length probability density, i.e. the second derivatives $\left[\gamma^{\prime \prime}(r)\right]$ of the relevant CFs $[\gamma(r)]$ have an algebraic form. The two solids are the regular tetrahedron (Ciccariello, 2005a) and the regular octahedron (Ciccariello, 2014). We have realized that these two second order derivatives can explicitly be twice integrated.

This short paper is devoted to report the resulting algebraic expressions, which are also of interest both in Rietveld analysis of wide angle diffraction patterns and in the stochastic geometry realm (Chiu et al. 2013) because particle isotropic covariograms are simply related to particle CFs.

\section{CF expressions}

To explain the derivation of these CFs it is sufficient to note that the key ingredient are: i) the integration by parts of each term contributing to $\gamma^{\prime \prime}(r)$ and having the form $f(r) \mathcal{T}(h(r))(\mathcal{T}$ being an inverse trigonometrical function), and ii) a convenient regrouping of the resulting terms. In fact,for some of these terms, after an integration by parts the resulting integrand $g(r) h^{\prime}(r) \mathcal{T}^{\prime}(h(r))$ [where $g(r)$ denotes a primitive of $f(r)$ ] has the form $R\left(r, P_{2}(r)^{1 / 2}\right)$, where $R(r, y)$ is a rational function and $P_{2}(x)$ a 2 nd degree polynomial. Thus, according to a general mathematical result [see, e.g., Caccioppoli, 1955], the primitive of $g(r) h^{\prime}(r) \mathcal{T}^{\prime}(h(r))$ has a simple algebraic form that is simply and safely worked out by MATHEMATICA. (The MATHEMATICA codes used to get the following results are found at http://digilander.libero.it/sciccariello/) In the other cases, say $\mathcal{T}_{1}(r), . ., \mathcal{T}_{4}(r)$, where $g_{i}(r) \mathcal{T}_{i}^{\prime}(r)$ has not the mentioned form, the mentioned property holds true after performing a suitable regrouping, say $g_{1}(r) \mathcal{T}_{1}^{\prime}(r)+g_{3}(r) \mathcal{T}_{3}^{\prime}(r)$ and $g_{2}(r) \mathcal{T}_{2}^{\prime}(r)+g_{4}(r) \mathcal{T}_{4}^{\prime}(r)$. 
It is now convenient to put

$$
\begin{gathered}
S_{\mathrm{T}} \equiv(3)^{1 / 2}, \quad V_{\mathrm{T}} \equiv 1 /\left[6(2)^{1 / 2}\right], \quad \alpha_{\mathrm{T}} \equiv \operatorname{arcos}(1 / 3), \\
S_{\mathrm{O}} \equiv 2(3)^{1 / 2}, \quad V_{\mathrm{O}} \equiv(2)^{1 / 2} / 3, \quad \alpha_{\mathrm{O}} \equiv \operatorname{arcos}(-1 / 3),
\end{gathered}
$$

where indices $\mathrm{T}$ and $\mathrm{O}$ respectively refer to the tetrahedron and the octahedron of unit sides, and

$$
\begin{gathered}
\Delta_{34}(r)(r) \equiv\left(4 r^{2}-3\right)^{1 / 2}, \quad \Delta_{11}(r)(r) \equiv\left(r^{2}-1\right)^{1 / 2} \\
\mathcal{T}_{1}(r) \equiv \frac{4 r^{4}-12 r^{2}+7}{4\left(1-r^{2}\right) \Delta_{34}(r)}, \quad \mathcal{T}_{2}(r) \equiv \frac{9 r^{2}-7}{3(3)^{1 / 2}\left(1-r^{2}\right) \Delta_{34}(r)}, \\
\mathcal{T}_{3}(r) \equiv \frac{2(2)^{1 / 2} r+3}{\Delta_{34}(r)}, \quad \mathcal{T}_{4}(r) \equiv \frac{(2)^{1 / 2} r}{\Delta_{34}(r)}, \quad \mathcal{T}_{5}(r) \equiv \frac{\Delta_{34}(r)}{2\left(1-r^{2}\right)}, \\
\mathcal{T}_{6}(r) \equiv \frac{\Delta_{34}(r)}{(3)^{1 / 2}}, \quad \mathcal{T}_{7}(r) \equiv \frac{6 r^{2}-5}{(3)^{1 / 2} \Delta_{34}(r)}, \quad \mathcal{T}_{8}(r) \equiv \frac{r\left(6 r^{2}-5\right)}{(2)^{1 / 2} \Delta_{34}(r)}, \\
\mathcal{T}_{9}(r) \equiv \frac{2 r^{4}+r^{2}-2}{2(2)^{1 / 2} r\left(1-r^{2}\right) \Delta_{34}(r)}, \quad \mathcal{T}_{10}(r) \equiv \frac{(3)^{1 / 2} \Delta_{34}(r)}{2 r^{2}-3}, \\
\mathcal{T}_{11}(r) \equiv \frac{\Delta_{34}(r)+1}{\Delta_{34}(r)-1}, \quad \mathcal{T}_{12}(r) \equiv \frac{2 r^{2}-3}{(3)^{1 / 2} \Delta_{34}(r)}, \quad \mathcal{T}_{13}(r) \equiv \frac{-2 r^{4}+12 r^{2}-9}{(3)^{1 / 2}\left(2 r^{2}-3\right) \Delta_{34}(r)} \\
\mathcal{T}_{14}(r) \equiv \frac{(3)^{1 / 2}\left(2 r^{8}-34 r^{6}+96 r^{4}-90 r^{2}+27\right)}{\left(10 r^{6}-54 r^{4}+72 r^{2}-27\right) \Delta_{34}(r)}, \quad \mathcal{T}_{15}(r) \equiv(3)^{1 / 2} \Delta_{11}(r) \\
\mathcal{T}_{16}(r) \equiv \frac{r}{(2)^{1 / 2} \Delta_{11}(r)}, \quad \mathcal{T}_{17}(r) \equiv \frac{7 r^{4}-4 r^{2}-4}{4(2)^{1 / 2} r\left(r^{2}-2\right) \Delta_{11}(r)}, \\
\mathcal{T}_{18}(r) \equiv \frac{\mathcal{T}_{19}(r) \equiv \frac{r^{2}+2 \Delta_{11}(r)-2}{2-r^{2}+2 \Delta_{11}(r)}}{3(3)^{1 / 2}\left(r^{2}-2\right) \Delta_{11}(r)}
\end{gathered}
$$

Then, one finds that the $\mathrm{CF}$ of the regular tetrahedron with unit sides is

\subsection{Tetrahedron CF}

$$
\begin{aligned}
\gamma_{\mathrm{T}, \mathrm{a}}(r) \equiv & 1-3 \sqrt{\frac{3}{2}} r+\frac{3\left(2^{3 / 2}+\pi-\alpha_{\mathrm{T}}\right) r^{2}}{\pi}-\frac{\left(6+5(3)^{1 / 2} \pi\right) r^{3}}{2^{5 / 2} \pi},(12 \\
\gamma_{\mathrm{T}, \mathrm{b}}(r) \equiv & \frac{3}{2^{5 / 2} r}-2-\frac{3\left((3)^{1 / 2}-3\right) r}{2^{1 / 2}}+\frac{3\left(2^{3 / 2}-\alpha_{T}-\pi\right) r^{2}}{\pi}- \\
& \frac{\left(6-12 \pi+5(3)^{1 / 2} \pi\right) r^{3}}{2^{5 / 2} \pi}
\end{aligned}
$$




$$
\begin{aligned}
\gamma_{\mathrm{T}, \mathrm{c}}(r) \equiv & \frac{9+8(3)^{1 / 2}}{12(2)^{1 / 2} r}-6+\frac{3\left(3+(3)^{1 / 2}\right) r}{2^{1 / 2}}+\frac{3\left(2^{3 / 2}-\alpha_{T}-3 \pi\right) r^{2}}{\pi}+ \\
& \frac{\left(12 \pi-6+(3)^{1 / 2} \pi\right) r^{3}}{2^{5 / 2} \pi} \\
\gamma_{\mathrm{T}, \mathrm{d}}(r) \equiv & \frac{9+8(3)^{1 / 2}}{24(2)^{1 / 2} r}+3+\frac{9\left(4+(3)^{1 / 2}\right) r}{2^{5 / 2}}+\frac{3 r^{2}\left(2^{3 / 2}-\pi-\alpha_{T}\right)}{\pi}-\frac{\left(3-12 \pi+(3)^{1 / 2} \pi\right) r^{3}}{2^{3 / 2} \pi} \\
& \frac{21 r \Delta_{34}(r)}{2^{3 / 2} \pi}+\frac{9}{12(2)^{1 / 2} \pi r}\left[\arctan \left(\mathcal{T}_{1}(\mathrm{r})\right)-\frac{8(3)^{1 / 2}}{9} \arctan \left(\mathcal{T}_{2}(\mathrm{r})\right)\right]+ \\
& \frac{3}{\pi}\left[\arctan \left(\mathcal{T}_{3}(\mathrm{r})\right)-3 \arctan \left(\mathcal{T}_{4}(\mathrm{r})\right)-4 \arctan \left(\frac{\mathcal{T}_{4}(\mathrm{r})}{2}\right)+\frac{1}{2} \arctan \left(\mathcal{T}_{5}(\mathrm{r})\right)\right] \\
& -\frac{3 r}{(2)^{1 / 2} \pi}\left[10 \arctan \left(\Delta_{34}(\mathrm{r})\right)+5(3)^{1 / 2} \arctan \left((3)^{1 / 2} \Delta_{34}(\mathrm{r})\right)+\arctan \left(\mathcal{T}_{5}(\mathrm{r})\right)-\right. \\
& \left.6(3)^{1 / 2} \arctan \left(\mathcal{T}_{6}(\mathrm{r})\right)+\frac{3^{1 / 2}}{2} \arctan \left(\mathcal{T}_{7}(\mathrm{r})\right)\right]+ \\
& \frac{6 r^{2}}{\pi}\left[\arctan \left(\mathcal{T}_{8}(\mathrm{r})\right)+\arctan \left(\mathcal{T}_{9}(\mathrm{r})\right)\right]-\frac{3 \mathrm{r}^{3}}{2 \pi} \sqrt{\frac{3}{2}}\left[\arctan \left(\mathcal{T}_{2}(\mathrm{r})\right)-\right. \\
& \left.\arctan \left(\mathcal{T}_{6}(\mathrm{r})\right)+2 \arctan \left(\mathcal{T}_{10}(\mathrm{r})\right)-\frac{8}{3^{1 / 2}} \arctan \left(\mathcal{T}_{11}(\mathrm{r})\right)\right]
\end{aligned}
$$

Here subscripts $a, b, c$, and $d$ respectively refer to the $r$ intervals $\left[0,1 /(2)^{1 / 2}\right]$, $\left[1 /(2)^{1 / 2}, \sqrt{2 / 3}\right],\left[\sqrt{2 / 3},(3)^{1 / 2} / 2\right]$ and $\left[(3)^{1 / 2} / 2,1\right]$ where the reported function definitions apply.

\subsection{Octahedron CF}

The expression of the $\mathrm{CF}$ of the regular octahedron with unit edges reads

$$
\begin{aligned}
& \gamma_{\mathrm{O}, \mathrm{a}}(r) \equiv 1-r\left(\frac{3}{2}\right)^{3 / 2}+\frac{3\left(\alpha_{O}-\pi+2(2)^{1 / 2}\right) r^{2}}{2 \pi}-\frac{\left(3-3 \pi+(3)^{1 / 2} \pi\right) r^{3}}{4(2)^{1 / 2} \pi}, \\
& \gamma_{\mathrm{O}, \mathrm{b}}(r) \equiv \frac{2}{r} \sqrt{\frac{2}{3}}-3-\frac{r}{2} \sqrt{\frac{3}{2}}+\frac{3 r^{2}\left(\alpha_{O}+\pi+2(2)^{1 / 2}\right)}{2 \pi}-\frac{\left(3-3 \pi+7(3)^{1 / 2} \pi\right) r^{3}}{4(2)^{1 / 2} \pi}
\end{aligned}
$$




$$
\begin{aligned}
\gamma_{\mathrm{O}, \mathrm{c}}(r) \equiv & \frac{2}{r} \sqrt{\frac{2}{3}}-3-\frac{r}{2} \sqrt{\frac{3}{2}}+\frac{3 r^{2}\left(\alpha_{O}+2(2)^{1 / 2}\right)}{2 \pi}-\frac{\left(36(1-\pi)+5(3)^{1 / 2} \pi\right) r^{3}}{48(2)^{1 / 2} \pi}- \\
& \frac{\left(17 r^{2}+3\right) \Delta_{34}(r)}{2(2)^{1 / 2} \pi r}+\frac{r}{\pi} \sqrt{\frac{3}{2}}\left[9 \arctan \left(\mathcal{T}_{6}(\mathrm{r})\right)+\arctan \left(3 \mathcal{T}_{6}(\mathrm{r})\right)\right]+ \\
& \frac{3 r^{2}}{\pi} \arctan \left(\frac{\mathrm{T}_{4}(\mathrm{r})}{2}\right)-\frac{\mathrm{r}^{3}}{8 \sqrt{6} \pi}\left[18 \arctan \left(\mathcal{T}_{6}(\mathrm{r})\right)+\right. \\
& 90 \arctan \left(\frac{1}{3 \mathcal{T}_{6}(\mathrm{r})}\right)+24 \arctan \left(\mathcal{T}_{7}(\mathrm{r})\right)-8 \arctan \left(\mathcal{T}_{12}(\mathrm{r})\right)- \\
& \left.\arctan \left(\mathcal{T}_{13}(\mathrm{r})\right)-6 \arctan \left(\mathcal{T}_{14}(\mathrm{r})\right)\right], \\
\gamma_{\mathrm{O}, \mathrm{d}}(r) \equiv & \frac{4(3)^{1 / 2} \pi-3}{3(2)^{1 / 2} \pi r}+3+\frac{\left(4(3)^{1 / 2} \pi-9\right) r}{3(2)^{1 / 2} \pi}+\frac{3 r^{2}}{4}-\frac{\left(6-3 \pi+4(3)^{1 / 2} \pi\right) r^{3}}{8(2)^{1 / 2} \pi}+ \\
& \frac{(2)^{1 / 2}}{\pi r}\left(1+2 r^{2}\right) \Delta_{11}(r)-\frac{2 \sqrt{6}}{\pi r} \arctan \left(\mathrm{T}_{15}(\mathrm{r})\right)- \\
& \frac{12}{\pi} \arctan \left(\mathrm{T}_{16}(\mathrm{r})\right)-\frac{2 \sqrt{6} \mathrm{r}}{\pi} \arctan \left(\mathrm{T}_{15}(\mathrm{r})\right)+\frac{3 \mathrm{r}^{2}}{2 \pi} \arctan \left(\mathcal{T}_{17}(\mathrm{r})\right)- \\
& \frac{r^{3}}{2(2)^{1 / 2} \pi}\left(2(3)^{1 / 2} \arctan \left(\mathcal{T}_{18}(\mathrm{r})\right)+3 \arctan \left(\mathcal{T}_{19}(\mathrm{r})\right)\right) . \\
&
\end{aligned}
$$

Subscripts subscripts $a, b, c$, and $d$ refer now to the r-intervals $[0, \sqrt{2 / 3}]$, $\left[\sqrt{2 / 3},(3)^{1 / 2} / 2\right],\left[(3)^{1 / 2} / 2,1\right]$ and $\left[1,(2)^{1 / 2}\right]$.

\section{Discussion}

It has been checked that the reported CF expressions obey the known constraints, i.e. $\gamma(0)=1, \gamma^{\prime}(0)=-S / 4 V, \gamma\left(D_{\max }\right)=\gamma^{\prime}\left(D_{\max }\right)=0, \quad 4 \pi \times$ $\int_{0}^{D_{\max }} r^{2} \gamma(r) d r=V$ and $4 \pi \int_{0}^{D_{\max }} r^{4} \gamma(r) d r=2 R_{G}{ }^{2} V, R_{\mathrm{G}}$ and $V$ denoting the Guinier giration radius and the volume of the particle.

A graphical comparison of the behaviours of the CFs of the first three Platonic solids, the sphere and the cylinder (of height equal to its diameter) is shown in Figure 1a. Using the property that $\gamma_{D_{\max }}(r)$, the $\mathrm{CF}$ of a given shape particle of maximal chord $D_{\max }$, is related to $\gamma(r)$, the CF of the particle with the same shape and unit maximal chord, as $\gamma_{D_{\max }}(r)=\gamma\left(r / D_{\max }\right)$, Figure 1 considers the case $D_{\max }=1$ for all the particles, so that the edge lengths of the tetrahedron, octahedron and cube are respectively equal to $1,2^{-1 / 2}$ and $3^{-1 / 2}$ while the sphere radius is $1 / 2$ and the cylinder height $2^{-1 / 2}$. It is noted that the choice of comparing particles of equal maximal chord is more reasonable than that of comparing particles of equal volume 
(see, e.g., Li et al., 2011) because the CF supports are equal. Fig. 1a shows that the CFs have a similar behaviour and that, passing from the tetrahedron to the octahedron, the cube and the cylinder, they approach that of the sphere. As $r$ approaches to one, the order is partly modified because the CF of the octahedron is smaller than the tetrahedron's (a feature not fully evident in the figure). This property is related to the number of vertex pairs that are distant $D_{\max }$ : this number is 6 for the octahedron and 12 for the tetrahedron. Figs. $1 \mathrm{~b}$ and $1 \mathrm{c}$ respectively plot the first and second derivative of the CFs considered in Fig. 1a. Passing from Fig.1a to Fig.1c, one sees that, as the derivative order $n$ increases, the behaviours of the $\gamma^{(n)}(r)$ s become more and more different in the five cases. One should in particular observe the first order discontinuities, located at $r=1 /(3)^{1 / 2}$ in the octahedron's and cube's case and at $r=1$ in the sphere's one, as well as the (infinitely high) cuspidal behaviour located at $r=1 /(2)^{1 / 2}$ for the cylinder's case. The finite discontinuities are generated by the elliptic parallelism (Ciccariello, 1985) of the polyhedron faces and the logarithmic one by the hyperbolic parallelism of the lateral surface of the cylinder (Ciccariello, 1989). The five intensities and their Porod plots are respectively shown in Fig. 2a and 2b. Note that in Fig. 2a the intensities have been scaled so as to make them equal at $q=0$. Fig. 2a shows that, as the face number of the polyhedra increases, the intensities approach that of the sphere. Fig. 2b, which refers to the non scaled intensities, reflects more details on the particle shapes, as the highest $S / V$ value of the tetrahedron, the presence of oscillations whose spacing is $2 \pi / d$ with $d$ equal to the distance between the parallel facets. In the tetrahedron case no parallelism is present and, consequently, the small oscillations fade away as $q$ increases. [In fact, they are related to the first sub-asymptotic term decreasing as $q^{-9 / 2}$ as it was shown by Ciccariello (2005b).] Finally Fig. 3 shows the effect of a Poisson size dispersion (Feigin \& Svergun, 1987), i.e. $p_{4,1}(d)=d^{4} e^{-d} / 4$ !. The intensities, smeared by this size dispersion, are still different even though their differences are reduced and the oscillations are completely washed out.

\section{Acknowledgment}

I am grateful to Dr. Wilfried Gille for his critical reading of the manuscript. 


\section{References}

Burger C. \& Ruland, W. (2001). Acta Cryst. A 57, 482-490.

Caccioppoli, R. (1956). Lezioni di Analisi Matematica, (Treves, Napoli, vol.II).

Chiu, S. N., Stoyan, D., Kendall, W.S. \& Mecke, J. (2013). Stochastic Geometry and its Applications, ( Wiley, Chichester, 3rd Ed.).

Ciccariello, S. (2014). J. Appl. Cryst. in the press.

Ciccariello, S. (2009). J. Math. Phys. 50, 103527(1-10).

Ciccariello, S. (2005b). Fibers $\&$ Textiles in East. Eur. 13, 41-46.

Ciccariello, S. (2005a). J. Appl. Cryst. 38, 97-106.

Ciccariello, S. (1989). Acta Cryst. A 45, 86-99.

Ciccariello, S. (1985). Acta Cryst. A 41, 560-568.

Debye, P. \& Bueche, A.M. (1949). J. Appl. Phys. 20, 518-525.

Feigin, L.A. \& Svergun, D.I. (1987). Structure Analysis by Small-Angle $X$-Ray $\&$ Neutron Scattering, (Plenum Press, New York).

Gille, W. (2013). Particle and Particle Systems characterization , (CRC Press, London)

Gille, W. (1999). J. Appl. Cryst. 32, 1100-1105.

Gille, W. (1987). Exp. Tech. Phys. 35, 93-99.

Goodisman, J. (1980). J. Appl. Cryst. 13, 132-135.

Guinier, A. \& Fournet, G. (1955). Small-Angle Scattering of X-rays, (Wiley, New York).

Li, X., Chewn, C. -Y., He, L., Meilleur, F., Myles, D.A.A., Liu, E., Zhang, Y., Smith, G.S., Herwig, K.W., Pynn, R. \& Chen, W.-R. (2011). J. Appl. Cryst. 44, 345-557. 

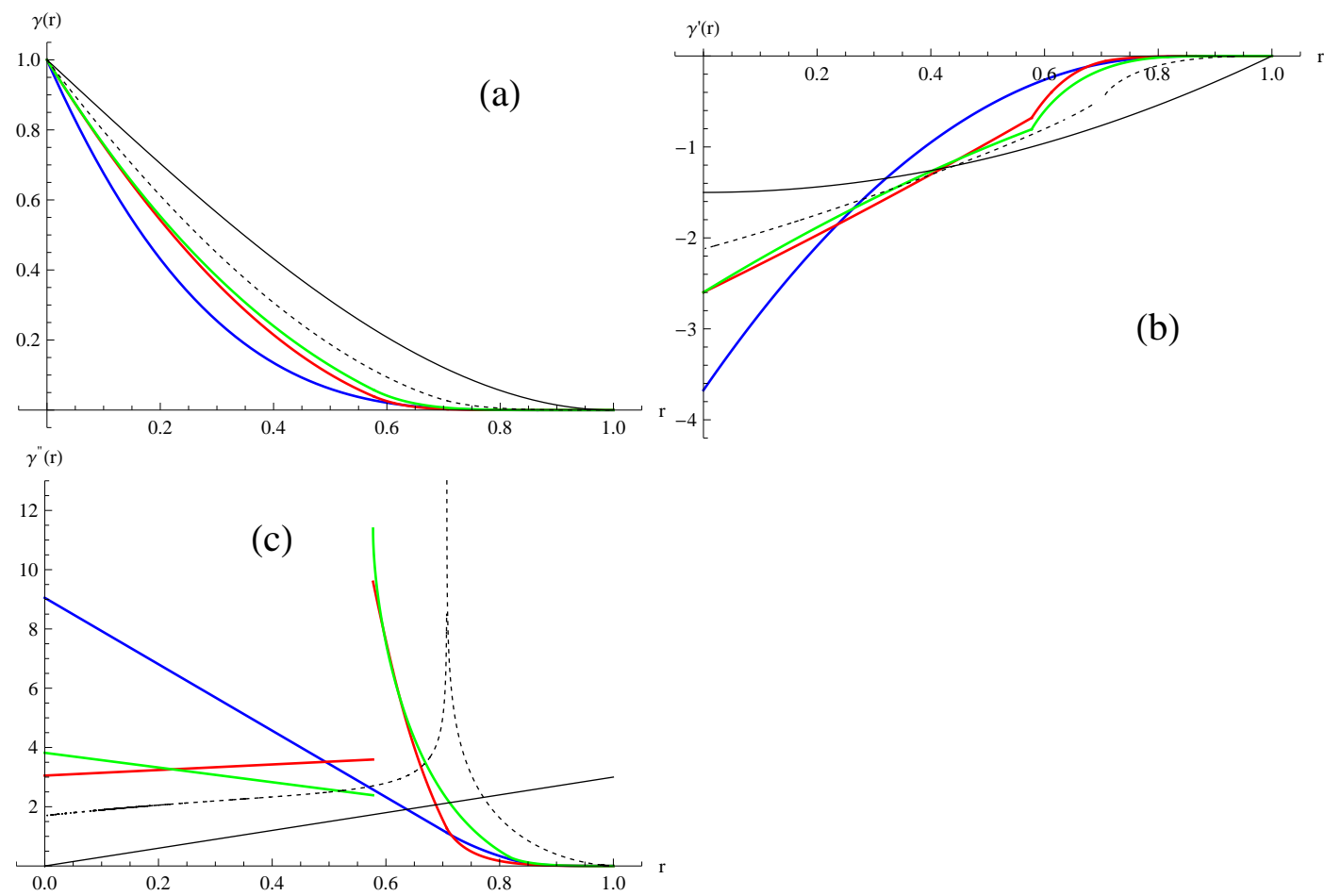

Figure 1: (a): Behaviour of the CFs of the tetrahedron (thick blue curve), octahedron (thick red), cube (thick green), cylinder (thin dotted black) and sphere (thin black). [All these solids have their largest diameter $D_{\text {max }}$, named $L$ in Figures 2 and 3, equal to one. Further, the height and the diameter of the cylinder are equal]; (b) behaviour of the first derivative of the CFs; (c): behaviour of the second derivative of the CFs 

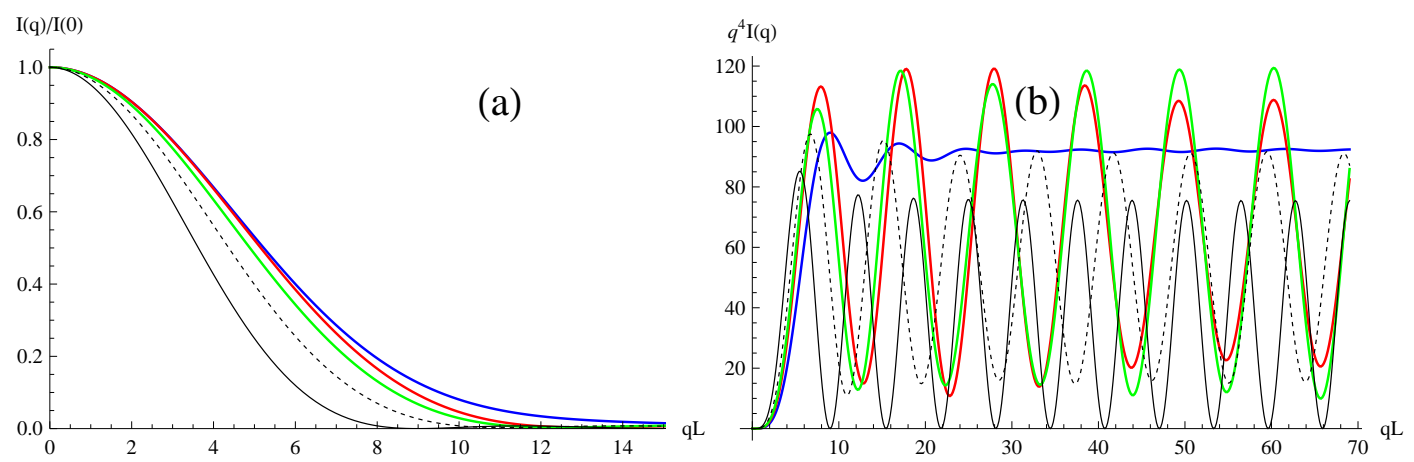

Figure 2: (a): Behaviour of the scattering intensities relevant to the solids considered in Fig.1 with the same colour meaning. Note that the intensities have been scaled to 1 at $q=0$; (b): Porod plot of the previous non-scaled intensities. $\left[L\right.$ is equal to $\left.D_{\max }.\right]$
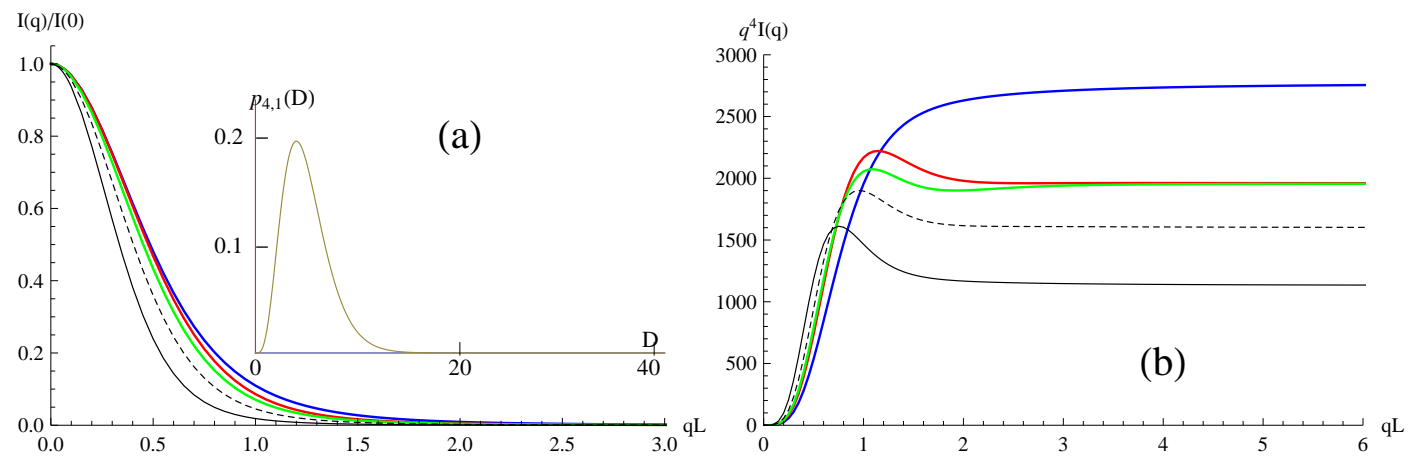

Figure 3: (a): Behaviour of the scattering intensities (scaled as in Fig.2a) relevant to polydisperse distributions of the particles considered in Fig.1. The particle size probability density is the Poisson one with $n=4$ and $\lambda=1$, i.e. $p_{4,1}(d)=d^{4} e^{-d} / 4$ ! and is shown in the inset; (b) Porod plot of the polydisperse non-scaled intensities. 